\title{
Oclusão da artéria central da retina secundária a cineangiocoronariografia via artéria braquial: relato de caso
}

\author{
Central retinal artery occlusion secondary \\ to brachial cineangiocoronography: case report
}

\author{
Augusto Nakashima ${ }^{1}$ \\ Alan Diego Negretto ${ }^{2}$ \\ Alexandre Antonio Marques Rosa ${ }^{3}$ \\ Yoshitaka Nakashima ${ }^{4}$ \\ Luciano Sólia Nasser ${ }^{5}$
}

\section{RESUMO}

Os autores relatam o caso de um paciente com insuficiência coronariana que desenvolveu quadro de oclusão de artéria central da retina após ser submetido a cateterização cardíaca por via braquial e realização de cineangiocoronariografia. Este procedimento pode desencadear fenômenos embólicos oculares consistentes com o quadro descrito.

Descritores: Cateterismo cardíaco/efeitos adversos; Embolia; Oclusão da artéria retiniana/ etiologia; Cineangiografia; Fatores de risco; Relatos de casos [Tipo de publicação]

\section{INTRODUÇÃO}

A cateterização cardíaca e a angiografia persistem como método ideal para avaliação da anatomia e fisiologia do coração e seus vasos. O primeiro experimento de cateterização cardíaca realizado em humanos data de 1929, tendo como objetivo principal a administração direta de drogas no tecido miocárdico ${ }^{(1)}$.

Com o desenvolvimento da abordagem técnica e do uso de contrastes para angiografia, este procedimento vem sendo amplamente utilizado. Atualmente, estima-se que, anualmente, mais de um milhão de pacientes sejam submetidos a cateterização cardíaca com angiografia, com propósitos diagnósticos e/ou terapêuticos ${ }^{(1)}$.

Como a cateterização cardíaca é um procedimento invasivo, não é surpresa que suas complicações potenciais incluam óbito, infarto miocárdico, acidente vascular cerebral, perfuração cardíaca ou de grandes vasos, e problemas vasculares locais. Os fenômenos embólicos secundários, bem estabelecidos em outras partes do organismo, são raramente manifestos na circulação retiniana ${ }^{(2)}$.

Neste artigo, relatamos o caso de uma oclusão da artéria central da retina $(\mathrm{OACR})$ secundária a cateterização cardíaca e angiografia.

\section{RELATO DE CASO}

VG, 55 anos, masculino, branco, encaminhado do Instituto do Coração (INCOR) do Hospital das Clínicas da Faculdade de Medicina da Universidade de São Paulo (HCFMUSP) para avaliação no ambulatório de oftalmologia do HCFMUSP, com história de baixa acuidade visual (A.V.) súbita em olho esquerdo, após ter sido submetido à cineangiocoronariografia via artéria braquial. Em relação aos antecedentes pessoais, apresentava histó- 
ria de insuficiência coronariana, em acompanhamento no INCOR. Sem antecedentes oculares pessoais prévios.

Ao exame oftalmológico, a A.V. era, com a melhor correção, OD: 20/20 e OE: percepção de movimentos de mão a 1 metro. Em olho esquerdo, havia um defeito pupilar aferente relativo (pupila de Marcus-Gunn). A biomicroscopia não mostrava alterações e a pressão intra-ocular era de $12 \mathrm{mmHg}$ no olho direito e $14 \mathrm{mmHg}$ no olho esquerdo.

A fundoscopia de olho direito não apresentava alterações, e no olho esquerdo notava-se uma palidez do disco óptico, com estreitamento arteriolar importante e a presença de coloração esbranquiçada da retina no pólo posterior, dando aspecto de mácula com mancha em cereja (Figura 1).

A angiofluoresceinografia retiniana do olho direito não apresentava anormalidades, e no olho esquerdo observava-se segmentação e interrupção do fluxo do contraste no interior da árvore arterial, e importante retardo no enchimento vascular retiniano.

\section{DISCUSSÃO}

A artéria central da retina é a principal fonte de irrigação sanguínea para a retina, nutrindo suas camadas mais internas ${ }^{(3)}$.

Em 1859, von Graefe descreveu uma obstrução embólica da artéria central da retina em paciente com endocardite e múltiplos êmbolos sistêmicos ${ }^{(4)}$. Após cinco anos, Sweiger descreveu todos os achados histopatológicos correspondentes a OACR ${ }^{(5)}$.

A OACR é um processo obstrutivo vascular raro e afeta pacientes por volta da sexta década de vida, sendo geralmente unilateral. Em geral, os pacientes queixam-se de uma perda visual indolor e súbita ${ }^{(6)}$. Um defeito pupilar aferente desenvolve-se segundos após a obstrução e persiste até que a obstrução se desfaça ${ }^{(7)}$.

$\mathrm{Na}$ fase aguda, a retina torna-se esbranquiçada devido a um edema intracelular da camada de fibras nervosas e gan-

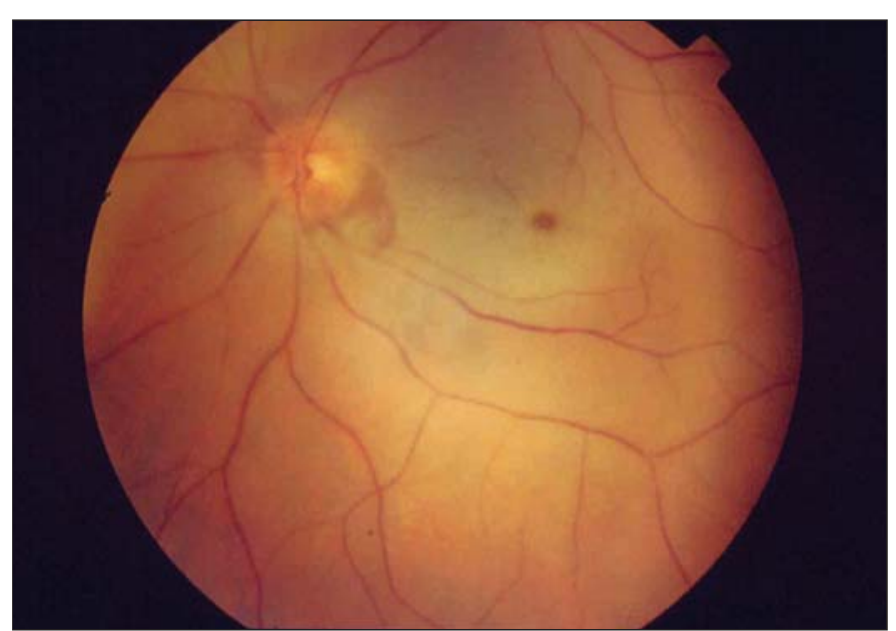

Figura 1 - Retinografia de olho esquerdo. Retina esbranquiçada no pólo posterior, devido à necrose esquêmica. Observar aspecto avermelhado da mácula (mácula cereja), típico de oclusão de artéria central da retina. glionares, sobretudo no pólo posterior, exceto na fóvea, dando o aspecto de mácula em mancha de cereja. Este fenômeno ocorre devido à retina ser fina na região foveolar, permitindo a visualização da circulação coróidea. Em casos graves de obstrução, há segmentação da coluna de sangue no interior dos vasos. Na maioria dos casos, o edema retiniano resolve-se em 4 a 6 semanas, deixando uma atrofia de nervo óptico, estreitamento arteriolar e diminuição da espessura da camada de fibras nervosas. A A.V. costuma variar de conta dedos a percepção de luz em $90 \%$ dos $\operatorname{casos}^{(7)}$.

Aproximadamente $25 \%$ dos olhos possuem uma artéria ciliorretiniana que supre parcial ou totalmente o feixe papilomacular e a fóvea. Se somente o feixe é preservado, a A.V. fica próxima de $20 / 100^{(8)}$. Em cerca de $10 \%$ dos olhos com OACR, a artéria ciliorretiniana nutre a fóvea e a A.V. fica próxima de 20/50 ou melhor, restando uma pequena ilha de visão central ${ }^{(8)}$.

Numerosas condições têm sido encontradas associadas à OACR da retina: A hipertensão arterial sistêmica é encontrada em aproximadamente $2 / 3$ dos pacientes, enquanto que $o$ diabetes melitus está presente em cerca de $1 / 4$ dos pacientes ${ }^{(9)}$. Essas duas condições constituem fatores de risco para a ocorrência de OACR. Elas são doenças que predispõem à formação de êmbolos. Nos casos de oclusões arteriais retinianas, o tipo de êmbolo mais comum é aquele proveniente dos depósitos ateroscleróticos das carótidas ${ }^{(10)}$. Podem se originar também do arco aórtico, de valvulopatias, de vegetações reumáticas, de injeções endógenas de coagulopatias, colagenoses e angioespasmos ${ }^{(11)}$.

Quanto à constituição dos êmbolos, o mais comum é formado por colesterol (placas de Hollenhorst), seguido pelo de plaquetas e por último os de cálcio ${ }^{(11)}$.

Um estudo multicêntrico (Retinal Emboli of Cardiac Origin Study Group- RECO) demonstrou a existência de doença estrutural cardíaca em aproximadamente $50 \%$ dos pacientes com oclusão da artéria central da retina, e apenas 10\% destes apresentavam patologia grave o suficiente para necessitarem de tratamento anticoagulante ou cirurgia cardíaca ${ }^{(12)}$. Aterosclerose das carótidas, na forma de uma placa ipsolateral ou estenose, é vista em $45 \%$ dos casos $^{(12)}$.

Um estudo semelhante, porém, usando ecocardiografia transesofágica detectou lesão cardíaca em $59 \%$ dos pacientes com OACR ${ }^{(13)}$.

O Beaver Dam Study, um estudo populacional $(n=4.926)$ recentemente observou a incidência de embolia retiniana em dez anos de seguimento da população local, encontrando uma taxa de $1,5 \%$ de oclusões embólicas nos pacientes de risco ${ }^{(14)}$.

A ocorrência de fenômenos embólicos é bem documentada na cateterização cardíaca seguida de angioplastia ${ }^{(15)}$. Dados de um estudo com 1.500 pacientes mostraram $21 \%$ de complicações após angioplastia coronária transluminal percutânea, incluindo 4 complicações neurológicas (1 acidente vascular cerebral e 3 ataques isquêmicos transitórios), porém sem nenhuma complicação ocular ${ }^{(15)}$. 
Alguns autores descrevem o caso de um paciente com retinopatia microembólica unilateral assintomática, um mês após angioplastia coronariana ${ }^{(16)}$. Outros autores relataram o caso de um paciente com angina pós infarto do miocárdio, que desenvolveu obstrução de um ramo da artéria central da retina após ser submetida a angioplastia coronariana transluminal percutânea ${ }^{(17)}$. Estes achados indicam que a cateterização cardíaca seguida de angioplastia pode produzir eventos embólicos em outros tecidos ${ }^{(17)}$.

A cateterização cardíaca seguida de angiografia oferece menor risco geral em relação à angioplastia, incluindo eventos embólicos. Este caso apresenta uma complicação embólica ocular (OACR) bastante rara, sendo também rara sua manifestação como decorrência de um procedimento de cateterização cardíaca sem angioplastia.

Há dois mecanismos de embolização potencial que podem explicar a oclusão de artéria central da retina neste paciente. Primeiro: liberação de debris ateroscleróticos originados de lesões vasculares pré-existentes. Este mecanismo pode ser decorrência direta da cateterização, pela ação da ponta do cateter (no caso da angioplastia, há risco adicional pela ação mais agressiva do balão); neste caso, geralmente há liberação de êmbolos de colesterol. Segundo: formação de trombos nos fios-guias, com embolização decorrente do processo de troca dos fios. Este mecanismo é bastante descrito em qualquer forma de cateterização cardíaca ${ }^{(17)}$. Sem a caracterização precisa do êmbolo como de colesterol, é impossível se determinar o mecanismo exato da embolização.

Lorentzen notou uma redução na sobrevida dos pacientes com OACR (5,5 anos) quando comparados com a expectativa da população normal $\left(15,4\right.$ anos) de mesma faixa etária ${ }^{(18)}$.

Portanto, o médico cardiologista deve estar ciente da possibilidade desta temível complicação, e encaminhar qualquer paciente com queixa visual após procedimento de cateterização cardíaca para realizar exame oftalmológico, o mais breve possível.

É importante salientar que o dano retiniano decorrente de uma OACR torna-se irreversível após 90 minutos ${ }^{(19)}$. Após 240 minutos ocorre total ou quase total atrofia do nervo óptico e da camada de fibras nervosas ${ }^{(20)}$. Por estas razões, preconiza-se investir em tratamento oftalmológico apenas se o paciente com OACR referir início da perda visual há menos de 24 horas ${ }^{(21)}$.

\section{ABSTRACT}

The authors report a case of a patient with coronary insufficiency who developed central retinal artery occlusion following cardiac catheterism through the brachial artery and cineangiocoronography. This procedure can lead to embolic phenomena like that which was reported.

Keywords: Heart catheterization/adverse effects; Embolism; Retinal artery occlusion/etiology; Cineangiography; Risk factors; Case reports [Publication type]

\section{REFERÊNCIAS}

1. Baim DS, Grossman W, editors. Cardiac catheterization, angiography, and intervention. $5^{\text {th }}$ ed. Baltimore, Williams \& Wilkins; 1996.

2. Johnson LW, Krone R. Cardiac catheterization 1991: a report of the Registry of the Society for Cardiac Angiography and Interventions (SCA\&I). Cathet Cardiovasc Diagn. 1993;28(3):219-20.

3. Tasman W, Jaeger E. Duane's Clinical Ophthalmology. Philadelphia: JB Lippincott; 1994.

4. von Graefe, A. Ueber Embolie der arteria centralis retinae als Urscahe plotzlicher erblingdung. Arch fur Ophthalmol. 1859;5:136-57.

5. Duke-Elder S, Dobree H. Diseases of retina. In: Duke-Elder S, Dobree H. System of ophthalmology. Saint Louis: Mosby; 1967. p.66-97.

6. Brown, GC, Magargal, LE. Central retinal artery obstruction and visual acuity. Ophthalmology. 1982;89(1):14-9.

7. Brown, GC, Shields, JÁ. Amaurosis fugax secondary to presumed cavernous hemangioma of the orbit. Ann Ophthalmol. 1981;13(1):1205-9.

8. Brown GC, Shields JÁ. Cilioretinal arteries and retinal arterial occlusion. Arch Ophthalmol. 1979;97(1):84-92.

9. Sharma S, Naqvi A, Sharma SM, Cruess AF, Brown GC. Transthoracic echocardiographic findings in patients with acute retinal arterial obstruction: a retrospective review. Retinal Emboli of Cardiac Origin Group. Arch Ophthalmol. 1996;114(10):1189-92, Comment in: Arch Ophthalmol. 1997;115(7):942.

10. Gold D. Retinal arterial occlusion. Trans Sect Ophthalmol Am Acad Ophthalmol Otolaryngol. 1977;83(3 Pt 1):OP392-408.

11. Abujamra S, Ávila M, Barsante C, Farah ME, Gonçalves JO, Lavinsky J, et al. Retina e vítreo: clínica e cirurgia. São Paulo: Roca; 2000.

12. Sharma S, Pater JL, Lam M, Cruess AF. Can different types of retinal emboli be realibly differentiated from one another? An inter-and intraobserver agreement study. Can J. Ophthalmol. 1998;33(3):144-8.

13. Inatomi $Y$, Hino $H$, Hashimoto $Y$, Furuyoshi N, Misumi I, Uchino M. Transesophageal echocardiography for detection of cardiac disease in patients with retinal artery occlusion. Intern Med. 2001;40(6):475-8.

14. Klein R, Klein BE, Moss SE, Meuer SM. Retinal emboli and cardiovascular disease. The Beaver Dam Eye study. Arch Ophthalmol. 2003:121(10):1446-51.

15. Dorros G, Cowley MJ, Simpson J, Bentivoglio LG, Block PC, Bourassa M, et al. Percutaneous transluminal coronary angioplasty: report of complications from the National Heart, Lung and Blood Institute PCTA Registry. Circulation. 1983;67(4):723-30.

16. Teitelbaum BA. Asymptomatic unilateral microembolic retinopathy secondary to percutaneous transluminal coronary angioplasty. J Am Optom Assoc. 1999;70(9):587-92.

17. Bucci FA Jr, Dimitsopulos TM, Krohel GB. Branch retinal artery occlusion secondary to percutaneous transluminal coronary angioplasty. Br J Ophthalmol. 1989;73(4):309-10.

18. Lorentzen SE. Occlusion of the central retinal artery: a follow-up. Acta Ophthalmol (Copenh). 1969;47(3):690-703.

19. Hayreh SS, Kolder HE, Weingeist TA. Central retinal artery occlusion and retinal tolerance time. Ophthalmology. 1980;87(1):75-8.

20. Hayreh SS, Jonas JB. Optic disk and retinal retinal nerve fiber layer damage after transient central retinal artery occlusion: an experimental study in rhesus monkeys. Am J Ophthalmol. 2000;129(6):786-95. Erratum in: Am J Ophthalmol. 2001;131(1):159.

21. Ryan SJ, editor. Retina. $5^{\text {th }}$ ed. St Louis: Mosby; 2006. 